\title{
Emission Policy in an Economic Union with Poisson Technological Change
}

\author{
Tapio Palokangas \\ University of Helsinki and HECER
}

Discussion Paper No. 160

April 2007

ISSN 1795-0562

HECER - Helsinki Center of Economic Research, P.O. Box 17 (Arkadiankatu 7), FI-00014 University of Helsinki, FINLAND, Tel +358-9-191-28780, Fax +358-9-191-28781, E-mail info-hecer@helsinki.fi, Internet www.hecer.fi 


\section{Emission Policy in an Economic Union with Poisson Technological Change}

\section{Abstract}

This study examines optimal emission policy in a union of countries. In each country, labor is allocated between production and R\&D which generates Poisson technological change. Production incurs emissions that are spread all over the union and aggravates pollution. Utility in any country depends negatively on both emissions and pollution in the union. The central planner of the union sets emission taxes for the countries. This study constructs a Pareto-optimal emission policy for the union.

JEL Classification: 041, H23, F15

Keywords: Emissions, Technological change, Economic Integration

Tapio Palokangas

Department of Economics

University of Helsinki

P.O. Box 17 (Arkadiankatu 7)

FI-00014 University of Helsinki

FINLAND

e-mail: tapio.palokangas@helsinki.fi 


\section{Introduction}

In this study, I examine the coordination of emission policy in a union of countries. The production of goods in any country incurs emissions that are spread all over the union, but efficiency in production can be improved by research and development $(R \& D)$. The outcome of $R \& D$ is random and follows a Poisson process. Welfare in any country depends positively on the level of consumption and negatively on total emissions in the union. The central planner of the union controls emissions by union-wide taxes. The purpose for study is to find out the Pareto-optimal emission taxes for the countries.

The impact of any environmental policy depends crucially on the existence of uncertainty. The papers [4], [8], [10] and [11] consider public policy by a growth model where productivity shocks follows a Wiener process. Soretz [9] applies that approach to environmental policy. In contrast, I assume that uncertainty is directly embodied in technological change in the form of Poisson processes.

Beltratti et al. [3] introduce a growth model where an environmental asset is a source of utility and depleted by a pollution process which is linked to consumption. They define the concept of the Green Golden rule as the best sustainable configuration, i.e. the path that gives the highest maintainable level of instantaneous utility. Ayong Le Kama [2] transforms this model by linking the pollution process to production. Following these papers, I search for the Green Golden for the economic union.

I assume that in each country there is a local planner that maximizes welfare and has enough instrument to control the allocation of resources in the country. The local planners of all countries realize that decisions in one country affect welfare in the other countries through emissions and pollution. Following Dixit [5], I assume that each local planner estimates the response of the others and makes its decisions by this information. To construct a Pareto optimal policy for the union, I introduce a central planner that maximizes welfare in the union through influencing the local planners' decisions by emission taxes.

This paper is organized as follows. Sections 2 and 3 present the general structure of the union and a single country. Sections 4 examine a local plan- 
ner's and section 5 the central planner's behavior. The optimization problems are solved by dynamic programming with Poisson jump processes (technological change) and an ordinary differential equation (the accumulation of pollution) as constraints.

\section{The union}

I consider a union of fixed number $n$ of similar countries. In country $j \in$ $\{1, \ldots, n\}$, there is a fixed labor supply $L$, of which the amount $l_{j}$ is used in production and the rest $z_{j}$ in $\mathrm{R} \& \mathrm{D}$ :

$$
L=l_{j}+z_{j}
$$

Emissions $m_{j}$ are in fixed proportion to labor input in production, $l_{j}$, in each country. By a proper choice of the unit of labor, local emissions $m_{j}$ and total emissions in the whole union, $m$, are then given by

$$
m_{j}=l_{j}, \quad m \doteq \sum_{j=1}^{n} m_{j}=\sum_{j=1}^{n} l_{j}
$$

Each country $j \in\{1, \ldots, n\}$ produces a different good. In the union, competitive firms produce a consumption good from all these goods according to

$$
\sum_{j=1}^{n} c_{j}=y=\prod_{j=1}^{n} y_{j}^{1 / n}
$$

where $c_{j}$ is consumption in country $j, y_{j}$ output in country $j$, and $y$ total consumption in the union. With some complication, the same results can be generalized for any neoclassical production function with constant returns to scale. Let $p_{j}$ be the price for good $j$. With Cobb-Douglas technology (3), the consumption price $p$ is obtained by minimizing the unit cost $\frac{1}{y} \sum_{j=1}^{n} p_{j} y_{j}=\sum_{j=1}^{n} p_{j} \frac{y_{j}}{y}$ of the consumption good by the input-output ratios $\left(y_{1} / y, \ldots, y_{n} / y\right)$ :

$$
p=\min _{y_{1} / y, \ldots, y_{n} / y}\left\{\sum_{j=1}^{n} p_{j} \frac{y_{j}}{y} \mid \prod_{j=1}^{n}\left(\frac{y_{j}}{y}\right)^{1 / n}=1\right\}=\prod_{j=1}^{n} p_{j}^{1 / n}
$$


Because in the model there is no money that would pin down the nominal price level at any time, the consumption price $p$ can be normalized at unity:

$$
1=p=\prod_{j=1}^{n} p_{j}^{1 / n} .
$$

Let $t$ be time and $P$ be the level of pollution in the union. Following [7], I assume that total emissions $m$ contribute the degree of pollution, $P$, but the nature absorbs pollution at a constant rate $h$ :

$$
\dot{P} \doteq d P / d t=m-h P
$$

\section{The countries}

Assume that all planners share the same preferences and that total emissions and the degree of pollution in the union decrease welfare in all countries. In country $j$, the utility from an infinite stream of its consumption $c_{j}$, emissions $m$ and pollution $P$ beginning at time $T$ is then given by

$$
E \int_{T}^{\infty} u\left(c_{j}, m, P\right) e^{-\rho(t-T)} d t, \quad \frac{\partial u}{\partial c_{j}}>0, \frac{\partial u}{\partial m}<0, \frac{\partial u}{\partial P}<0,
$$

where $E$ is the expectation operator, $\rho>0$ the constant rate of time preference and $u$ the level of instantaneous utility. Because it is impossible to find any analytical solution for the general case (6) in Bellman's dynamic programming, I specify the instantaneous utility function in the exponential form

$$
u\left(c_{j}, m, P\right) \doteq c_{j}^{\sigma} m^{-\delta} P^{-\nu}, \quad 0<\sigma<1, \delta>0, \quad \nu \geq 0,
$$

where $\sigma, \delta$ and $\nu$ are constants. The constant $1-\sigma \in(0,1)$ is the constant rate of risk aversion. Following [2] and [3], I make the following definition:

Definition The Green Golden Rule $(G G R)$ is the pattern of development that gives the highest maintainable level of instantaneous utility (7) for all countries $j \in\{1, \ldots, n\}$.

The efficiency of labor $l_{j}$ in production in country $j$ is $A^{\gamma_{j}}$, where $A>1$ is a constant and $\gamma_{j}$ is the serial number of technology. In the advent of 
technological change in country $j$, this efficiency increases from $A^{\gamma_{j}}$ to $A^{\gamma_{j}+1}$. Total output in country $j$ is given by

$$
y_{j}=A^{\gamma_{j}} l_{j}
$$

In production, firms employ labor $l_{j}$ up to the point where the wage $w_{j}$ is equal to the output price $p_{j}$ times the marginal product of labor, $\partial y_{j} / \partial l_{j}$ :

$$
w_{j}=p_{j} \partial y_{j} / \partial l_{j}=p_{j} A^{\gamma_{j}}
$$

The improvement of technology in country $j$ depends on the labor $z_{j}$ devoted to R\&D. I assume that in a small period of time $d t$, the probability that R\&D leads to development of a new technology is given by $\lambda z_{j} d t$, while the probability that $R \& D$ remains without success is given by $1-\lambda z_{j} d t$, where $\lambda$ is the productivity of labor in R\&D. This defines a Poisson process $q$ with

$$
d q_{j}= \begin{cases}1 & \text { with probability } \lambda z_{j} d t \\ 0 & \text { with probability } 1-\lambda z_{j} d t\end{cases}
$$

where $d q_{j}$ is the increment of the process $q_{j}$.

I assume that the central planner imposes a uniform $\operatorname{tax} \tau$ on the product $w_{j} m_{j}$ of wages $w_{j}$ and emissions $m_{j}$, and pays a uniform subsidy $b$ to labor income $w_{j} L$ throughout all countries $j$ in the union. Thus, given (2), (8) and (9), consumption in country $j$ is determined by

$$
c_{j}=p_{j} y_{j}-\tau w_{j} m_{j}+b w_{j} L=p_{j} A^{\gamma_{j}}\left[(1-\tau) l_{j}+b L\right],
$$

where $p_{j} y_{j}$ is income from production, $\tau w_{j} m_{j}$ emission taxes and $b w_{j} L$ subsidies for labor. Noting (2), the central planner's budget constraint is then

$$
b \sum_{j=1}^{n} w_{j} L=\tau \sum_{j=1}^{n} w_{j} m_{j}=\tau \sum_{j=1}^{n} w_{j} l_{j},
$$

where $b \sum_{j=1}^{n} w_{j} L$ is subsidies and $\tau \sum_{j=1}^{n} w_{j} m_{j}$ emission taxes in the union. In line with [5], I assume on the planners' strategic behavior the following:

Assumption Planner $j \in\{1, \ldots, n\}$ forms expectations on the prospective responses of the other local planners $\ell \neq j$ to its action. It anticipates the 
others $\ell \neq j$ to increase their emissions $m_{\ell}$ by the constant $\beta \in(-\infty, 1)$ units after it itself has increased its emissions $m_{j}$ by one unit.

This assumption and (2) imply $d l_{\ell} / d l_{j}=d m_{\ell} / d m_{j}=\beta$ for all $\ell \neq j$ and

$$
m=M\left(l_{j}, n, \beta\right) \text { with } \frac{\partial M}{\partial l_{j}}=1+\sum_{\ell \neq j} \frac{d l_{\ell}}{d l_{j}}=1+(n-1) \beta .
$$

Here, $M\left(l_{j}, n, \beta\right)$ is planner $j$ 's perceived supply function of total emissions in the union. Because in the model all local planners $j=1, \ldots, n$ are in symmetric position, they have the same perceived supply function (13). A local planner takes $\beta$ as a constant, but at the level of the whole union the parameter $\beta$ adjusts to keep the perceived supply of emissions, $M\left(l_{j}, n, \beta\right)$, equal to actual emissions $m$ for all planners $j \in\{1, \ldots, n\}$.

\section{The local planners}

Noting (13) and (5), local planner $j$ 's perceived pollution evolves according to

$$
\dot{P}=M\left(l_{j}, n, \beta\right)-h P, \quad \partial \dot{P} / \partial l_{j}=\partial M / \partial l_{j}=1+(n-1) \beta .
$$

Local planner $j$ maximizes the utility of country $j$, (6) with (7), by consumption $c_{j}$ and labor input $\left(l_{j}, z_{j}\right)$ subject to the resource constraint (1), Poisson technological change (10), the budget constraint (11), expectations (13) and pollution (14), on the assumption that the price $p_{j}$, the tax $\tau$, the subsidy $b$ and the parameter $\beta$ are kept constant. The value of the optimal program for planner $j$ starting at time $T$ is then given by

$$
\begin{aligned}
& \Gamma^{j}\left(\gamma_{j}, P, \tau, b, p_{j}, \beta, n, T\right) \doteq \max _{\left(c_{j}, l_{j}, z_{j}\right) \text { s.t. }(1),(10),(11),(13),(14)} E \int_{T}^{\infty} \frac{c_{j}^{\sigma}}{m^{\delta}} P^{-\nu} e^{-\rho(t-T)} d t \\
& =\max _{\left(l_{j}, z_{j}\right) \text { s.t. }(1),(10),(14)} E \int_{T}^{\infty} \frac{p_{j}^{\sigma}}{P^{\nu}} A^{\sigma \gamma_{j}} \frac{\left[(1-\tau) l_{j}+b L\right]^{\sigma}}{M\left(l_{j}, n, \beta\right)^{\delta}} e^{-\rho(t-T)} d t
\end{aligned}
$$

I denote $\Gamma^{j}=\Gamma^{j}\left(\gamma_{j}, P, \tau, b, p_{j}, \beta, n, T\right)$ and $\widetilde{\Gamma}^{j}=\Gamma^{j}\left(\gamma_{j}+1, P, \tau, b, p_{j}, \beta, n, T\right)$. The Bellman equation corresponding to the optimal program (15) is given by

$$
\rho \Gamma^{j}=\max _{l_{j}} \Psi^{j}\left(l_{j}, \gamma_{j}, P, \tau, b, p_{j}, \beta, n, T\right),
$$


where

$$
\begin{gathered}
\Psi^{j}\left(l_{j}, \gamma_{j}, P, \tau, b, p_{j}, \beta, n, T\right)=\frac{p_{j}^{\sigma}}{P^{\nu}} A^{\sigma \gamma_{j}} \frac{\left[(1-\tau) l_{j}+b L\right]^{\sigma}}{M\left(l_{j}, n, \beta\right)^{\delta}}+\frac{\partial \Gamma^{j}}{\partial P} \dot{P} \\
+\lambda\left(L-l_{j}\right)\left[\widetilde{\Gamma}^{j}-\Gamma^{j}\right] .
\end{gathered}
$$

This, (13) and (14) lead to the first-order condition

$$
\begin{aligned}
& \frac{\partial \Psi^{j}}{\partial l_{j}}=\left[\frac{(1-\tau) \sigma}{(1-\tau) l_{j}+b L}-\frac{\delta}{M} \frac{\partial M}{\partial l_{j}}\right] \frac{p_{j}^{\sigma}}{P^{\nu}} A^{\sigma \gamma_{j}} \frac{\left[(1-\tau) l_{j}+b L\right]^{\sigma}}{M\left(l_{j}, n, \beta\right)^{\delta}}+\frac{\partial \Gamma^{j}}{\partial P} \frac{\partial \dot{P}}{\partial l_{j}} \\
& =\left\{\frac{(1-\tau) \sigma}{(1-\tau) l_{j}+b L}-\frac{\delta}{m}[1+(n-1) \beta]\right\} \frac{p_{j}^{\sigma}}{P^{\nu}} A^{\sigma \gamma_{j}} \frac{\left[(1-\tau) l_{j}+b L\right]^{\sigma}}{M\left(l_{j}, n, \beta\right)^{\delta}} \\
& \quad+\frac{\partial \Gamma^{j}}{\partial P}[1+(n-1) \beta]-\lambda\left[\widetilde{\Gamma}^{j}-\Gamma^{j}\right]=0 .
\end{aligned}
$$

To solve the dynamic program, I try the solution that the value of the program, $\Gamma^{j}$, is in fixed proportion $\vartheta_{j}>0$ to instantaneous utility (7):

$$
\Gamma^{j}\left(\gamma_{j}, P, \tau, b, p_{j}, \beta, n, T\right)=\vartheta_{j} p_{j}^{\sigma} A^{\sigma \gamma_{j}}\left[(1-\tau) l_{j}+b L\right]^{\sigma} m^{-\delta} P^{-\nu} .
$$

This implies

$$
\widetilde{\Gamma}^{j} / \Gamma^{j}=A^{\sigma}, \quad \partial \Gamma^{j} / \partial P=-\nu \Gamma^{j} / P .
$$

Inserting (19) and (20) into the Bellman equation (16) and (17) yields

$$
1 / \vartheta_{j}=\rho+\left(1-A^{\sigma}\right) \lambda\left(L-l_{j}\right)+\nu \dot{P} / P>0 .
$$

Inserting (19), (20) and (21) into the first-order condition (18) yields

$$
\begin{aligned}
& \left(A^{\sigma}-1\right) \lambda+\frac{\nu}{P}[1+(n-1) \beta]=\left\{\frac{(1-\tau) \sigma}{(1-\tau) l_{j}+b L}+\frac{\delta}{m}[1+(n-1) \beta]\right\} \frac{1}{\vartheta_{j}}= \\
& \left\{\frac{(1-\tau) \sigma}{(1-\tau) l_{j}+b L}-\frac{\delta}{m}[1+(n-1) \beta]\right\}\left[\rho+\left(1-A^{\sigma}\right) \lambda\left(L-l_{j}\right)+\nu \frac{\dot{P}}{P}\right] .
\end{aligned}
$$

Because there is symmetry throughout countries $j=1, \ldots, n$ in the model, noting (1), (2), (4) and (9), it is true that

$$
l_{j}=l, \quad z_{j}=z=L-l, c_{j}=c, \quad M=m=n l, p_{j}=p=1, \quad w_{j}=A^{\gamma_{j}} .
$$


Given (3), (8) and (23), the consumption good is produced according to

$$
c_{j}=c=\frac{y}{n}=\frac{1}{n} \prod_{j=1}^{n} y_{j}^{1 / n}=\frac{1}{n} A^{\gamma} \prod_{j=1}^{n} l_{j}^{1 / n}=\frac{l}{n} A^{\gamma}, \quad \gamma \doteq \frac{1}{n} \sum_{j=1}^{n} \gamma_{j},
$$

where $\gamma$ the serial number of the consumption-good technology. Because the improvement of productivity $A^{\gamma_{j}}$ in the production of good $j$, (8), follows the Poisson process (10), and because labor inputs $l_{j}$ are constant over time in the stationary state, then, given (3), the improvement of productivity in the production $y$ of the consumption good follows the Poisson process $q$ with

$$
d q= \begin{cases}1 & \text { with probability } \lambda(L-l) d t \\ 0 & \text { with probability } 1-\lambda(L-l) d t .\end{cases}
$$

Thus, the expected growth rate of consumption $y$ in the stationary state is

$$
g \doteq E\left[\log A^{\gamma+1}-\log A^{\gamma}\right]=(\log A) \lambda z=(\log A) \lambda(L-l)
$$

where $E$ is the expectation operator (cf., [1], p. 59, and [12]). Noting (23), the budget constraint (12) changes into

$$
b=\tau \sum_{j=1}^{n} w_{j} l_{j} /\left(L \sum_{j=1}^{n} w_{j}\right)=\frac{\tau l}{L} .
$$

Finally, given (23), the accumulation of pollution (14) changes into

$$
\dot{P}=n l-h P \quad \text { with } \quad \partial \dot{P} / \partial l=n .
$$

I consider the stationary state where the union has attained its equilibrium level of pollution. Given (28), the conditions for that state are

$$
\dot{P}=0, \quad h P=M(l)=m=n l .
$$

Inserting (23), (27) and (29) into (22) and solving for $l$, one obtains the equilibrium level of emissions in the stationary state:

$$
\begin{aligned}
l= & \frac{1 / n+(1-1 / n) \beta}{(1-\tau) \sigma-\delta[1 / n+(1-1 / n) \beta]-1} \frac{\nu h / \lambda}{A^{\sigma}-1} \\
& \quad+\frac{(1-\tau) \sigma-\delta[1 / n+(1-1 / n) \beta]}{1-(1-\tau) \sigma+\delta[1 / n+(1-1 / n) \beta]}\left(\frac{\rho / \lambda}{A^{\sigma}-1}-L\right) .
\end{aligned}
$$




\section{The central planner}

Noting (2) and (24), the welfare in the union takes the form

$$
U(c, m, T)=\int_{T}^{\infty} A^{\sigma \gamma} n^{-\sigma-\delta} l^{\sigma-\delta} P^{-\nu} e^{-\rho(t-T)} d t
$$

The central planner maximizes this welfare by labor in production, $l$, subject to technological change (25) and the dynamics of pollution (28). Noting (24), the value of the optimal program starting at time $T$ for the central planner is

$$
\Gamma(\gamma, P, T)=\max _{l \text { s.t. }(25),(14)} E \int_{T}^{\infty} A^{\sigma \gamma} n^{-\sigma-\delta} l^{\sigma-\delta} P^{-\nu} e^{-\rho(t-T)} d t .
$$

The Bellman equation corresponding to this optimal program is given by

$$
\begin{aligned}
& \rho \Gamma(\gamma, P, T) \\
& =\max _{l}\left\{\frac{A^{\sigma \gamma}}{P^{\nu}} \frac{l^{\sigma-\delta}}{n^{\sigma+\delta}}+\frac{\partial \Gamma}{\partial P} \dot{P}+\lambda(L-l)[\Gamma(\gamma+1, P, T)-\Gamma(\gamma, P, T)]\right\} .
\end{aligned}
$$

Noting (28), this maximization leads to the first-order condition:

$$
\frac{\partial\{\}}{\partial l}=(\sigma-\delta) \frac{A^{\sigma \gamma}}{P^{\nu}} \frac{l^{\sigma-\delta-1}}{n^{\sigma+\delta}}+n \frac{\partial \Gamma}{\partial P}-\lambda[\Gamma(\gamma+1, P, T)-\Gamma(\gamma, P, T)]=0 .
$$

Noting $\sigma<1$, the second-order condition $\partial^{2}\{\} / \partial l^{2}<0$ is equivalent to $\sigma>\delta$.

To solve the dynamic program, I try the solution that the value of the program, $\Gamma$, is in fixed proportion $\vartheta>0$ to instantaneous utility:

$$
\Gamma(\gamma, T)=\vartheta A^{\sigma \gamma} n^{-\sigma-\delta} l^{\sigma-\delta} P^{-\nu} .
$$

This implies

$$
\Gamma(\gamma+1, T) / \Gamma(\gamma, T)=A^{\sigma}>1, \quad \partial \Gamma / \partial P=-\nu \Gamma / P<0 .
$$

From $\partial \Gamma / \partial P<0$ and the first-order condition (34) it follows that $\sigma>\delta$.

I consider this equilibrium only in the stationary state where $\dot{P}=0$ and (29) hold true. Inserting (35) and (36) into the Bellman equation (33) yields

$$
1 / \vartheta=\rho+\left(1-A^{\sigma}\right) \lambda(L-l)>0 .
$$


Inserting (29), (35), (36) and (37) into (34) and solving for $l$, one obtains the Pareto-optimal level of emissions, $l^{*}$ :

$$
l^{*} \doteq \frac{\left(A^{\sigma}-1\right)^{-1}}{(1-\sigma+\delta) \lambda}\left\{(\delta-\sigma)\left[\rho+\left(1-A^{\sigma}\right) L\right]-h \nu\right\}, \quad \frac{\partial l^{*}}{\partial(h \nu)}<0 .
$$

This, $l^{*}>0, \sigma>\delta, \sigma<1$ and $A^{\sigma}>1$ yield $\rho+\left(1-A^{\sigma}\right) L<0$ and $\partial l^{*} / \partial \delta<0$. Noting this, (26) and (38), the Pareto-optimal growth is $g^{*}=(\log A)\left(L-l^{*}\right)$, for which $\partial g^{*} / \partial(h \nu)>0$ and $\partial g^{*} / \partial \delta>0$. I summarize the result as follows:

Proposition 1 The Pareto-optimal growth rate $g^{*}$ is the higher, the more emissions or pollution are disliked (i.e. the bigger $\delta$ or $\nu$ ), or the higher the absorbtion rate of pollution, $h$.

When emissions or pollution is disliked, labor is transferred from "dirty" production to "clean" R\&D, which boosts economic growth. A high absorbtion rate eases the constraint for the central planner and boosts economic growth.

The central planner sets the tax parameter $\tau$ to establish the Pareto optimum $l=l^{*}$. From $\rho+\left(1-A^{\sigma}\right) \lambda L<0,(30)$ and (38) it then follows that

$$
\begin{aligned}
& \tau^{*} \doteq \frac{1-\beta}{\sigma}\left(1-\frac{1}{n}\right) \frac{\delta+(1-\sigma) h \nu /\left[\rho+\left(1-A^{\sigma}\right) \lambda L\right]}{1-h \nu /\left[\rho+\left(1-A^{\sigma}\right) \lambda L\right]}>0, \quad \frac{\partial \tau^{*}}{\partial(h \nu)}<0 \\
& \frac{\partial \tau^{*}}{\partial \delta}=\frac{1-\beta}{\sigma}\left(1-\frac{1}{n}\right) \frac{1}{1-h \nu /\left[\rho+\left(1-A^{\sigma}\right) \lambda L\right]}>0
\end{aligned}
$$

Thus, the following result is obtained:

Proposition 2 The optimal emission tax $\tau^{*}$ that leads to the Green Golden Rule is given by (39). This increases with the number $n$ of countries. When the union is a single jurisdiction, $n=1$, the tax is zero. The tax is the lower, the greater is the other local planners' anticipated response $\beta$. The more emissions are disliked (i.e. the higher $\delta$ ), the less pollution is disliked (i.e. the smaller $\nu$ ) or the smaller the absorbtion rate of pollution, $h$, the higher the emission tax.

The more countries in the union (i.e. the bigger $n$ ), the higher proportion of the emissions of a country falls outside the country and the less a local planner is willing to reduce emissions. Consequently, a higher tax is needed to 
make a local planner to reduce its emissions. Noting (13) and the symmetry $l_{j} / m \approx 1 / n$, the elasticity of total emissions $m$ with respect to emissions in a single country $l_{j}$ is given by

$$
\frac{l_{j}}{m} \frac{\partial M}{\partial l_{j}}=[1+(n-1) \beta] \frac{l_{j}}{m} \approx \frac{1}{n}+\left(1-\frac{1}{n}\right) \beta .
$$

With estimating this elasticity, one can estimate $\beta$. The more a local planner expects the others to follow its policy (i.e. the bigger $\beta$ ), the less space it has for raising its emissions and the smaller tax (39) is adequate for reducing its emissions. Disliking emissions (i.e. a high $\delta$ ) increases the benefit of the emission tax, $\partial \tau^{*} / \partial \delta>0$. Disliking pollution (i.e. a high $\nu$ ) or high absorbtion rate $h$ strengthens the welfare effect of the tax. In that case, a smaller $\operatorname{tax} \tau^{*}$ is adequate for maintaining the Pareto optimum, $\partial \tau^{*} / \partial(h \nu)<$ 0 .

\section{Conclusions}

This study examines optimal emission policy in a union of countries. The results are expressed in the form of Pareto-optimal emission taxes. These lead to the best sustainable configuration (e.g. the Green Golden Rule) in the stationary state. The optimal emission tax can be determined once the response parameter $\beta$ is estimated. The other relevant information for the tax contains the wages, the parameters of the utility function and the absorbtion rate of pollution. The model can be extended in the following directions:

1. Countries can be of different size. A big country is a Stackelberg leader, which takes the optimal responses of smaller countries into account.

2. There is technological diffusion so that investment in R\&D in one country improves productivity also in the other countries.

3. There is capital that is accumulated by private saving. This forms a differential equation that enters as an constraint in the optimization. Emissions can be complementary to labor, capital or output.

4. Following [4], [9], [10] and [11], productivity can follow a Wiener process, which means that it is a Poisson-Wiener process that is optimized. 


\section{References}

[1] P. Aghion and P. Howitt: Endogenous Growth Theory, MIT Press (Cambridge, Massachusetts, 1998).

[2] Ayong Le Kama, A., Sustainable growth, renewable resources and pollution, 2001, Journal of Economic Dynamics and Control, 25, 1911-1918.

[3] Beltratti, A., Chichilnisky, G., \& Heal, G.M., Sustainable growth and the green golden rule, in I. Goldin \& L.A. Winters: The Economics of Sustainable Development, Cambridge University Press (Cambridge, United Kingdom, 1994).

[4] Corsetti, G., A portfolio approach to endogenous growth: equilibrium and optimal policy, 1997, Journal of Economic Dynamics and Control, $21,1627-1644$.

[5] Dixit, A., Comparative statics for oligopoly, 1986, International Economic Review, 27, 107-122.

[6] A. Dixit,, \& K. Pindyck: Investment under Uncertainty, Princeton University Press (Princeton, 1994).

[7] Michel, P., \& Rotillon, G., Disutility of pollution and endogenous growth, 1995, Environmental and Resource Economics, 6, 25-51.

[8] Smith, W.T., Feasibility and transversality conditions for models of portfolio choice with non-expected utility in continuous time, 1996, Economic Letters, 53, 123-131.

[9] Soretz, S., Stochastic pollution and environmental care in an endogenous growth model, 2003, The Manchester School, 71, 448-469.

[10] Turnovsky, S.J.: Methods of Macroeconomic Dynamics, MIT Press (Cambridge, Massachusetts, 1995)

[11] Turnovsky, S.J., On the role of government in a stochastically growing economy, 1999, Journal of Economic Dynamics and Control, 104, 275298.

[12] Wälde, K., Optimal saving under Poisson uncertainty, 1999, Journal of Economic Theory, 87, 194-217. 